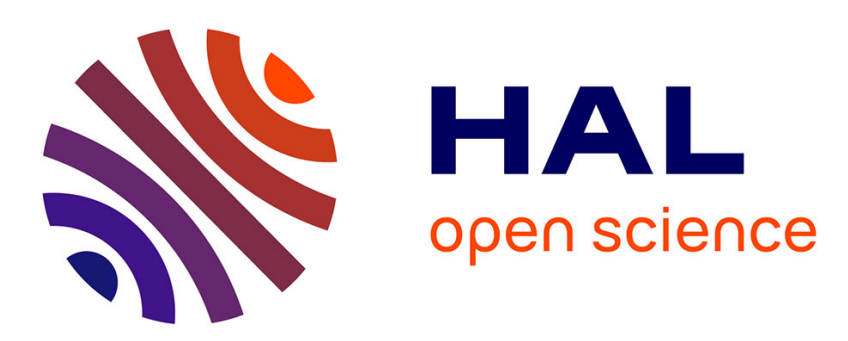

\title{
Local search proximal algorithms as decision dynamics with costs to move
}

\author{
Hedy Attouch, Antoine Soubeyran
}

\section{To cite this version:}

Hedy Attouch, Antoine Soubeyran. Local search proximal algorithms as decision dynamics with costs to move. Set Valued Analysis, 2010, 19 (1), pp.157-177. 10.1007/s11228-010-0139-7 . hal-00803790

\section{HAL Id: hal-00803790 \\ https://hal.science/hal-00803790}

Submitted on 22 Mar 2013

HAL is a multi-disciplinary open access archive for the deposit and dissemination of scientific research documents, whether they are published or not. The documents may come from teaching and research institutions in France or abroad, or from public or private research centers.
L'archive ouverte pluridisciplinaire HAL, est destinée au dépôt et à la diffusion de documents scientifiques de niveau recherche, publiés ou non, émanant des établissements d'enseignement et de recherche français ou étrangers, des laboratoires publics ou privés. 


\title{
Local search proximal algorithms as decision dynamics with costs to move
}

\author{
H. ATTOUCH* ${ }^{* \dagger}$ A. SOUBEYRAN ${ }^{\ddagger}$
}

April, 12, 2010

\begin{abstract}
Acceptable moves for the "worthwhile-to-move" incremental principle are such that "advantages to move" are higher than some fraction of "costs-to-move". When combined with optimization, this principle gives raise to adaptive local search proximal algorithms. Convergence results are given in two distinctive cases, namely low local costs-to-move and high local costs-to-move. In this last case, one obtains a dynamic cognitive approach to Ekeland's $\epsilon$-variational principle. As a general property, introduction of costs-to-move in the algorithms yields robustness and stability properties.
\end{abstract}

2000 Mathematics Subject Classification: 49M37, 90B50, 90C25, 90C30, 90C31, 90C56, 91E10, 91E40, 91E45.

Key words: costs-to-move, decision dynamics, exploration process, friction, inertia, local optimization, local search algorithms, proximal algorithms, worthwhile-to-move incremental process.

\section{Introduction: decision dynamics with costs to move and optimization}

In this paper, we explore the relationship between decision processes with costs to move and optimization algorithms. This will lead us to introduce a new class of algorithms, the so-called local search proximal algorithms. In this context, the regularizing term of the proximal algorithm can be interpreted as a cost to move. This approach considerably enlarges the traditional framework of proximal algorithms, and puts to the fore numerous aspects directly linked to decision, and cognitive sciences. We shall pay particular attention to the following aspects, which are relevant both of decision sciences

*I3M UMR CNRS 5149, Université Montpellier II, Place Eugène Bataillon, 34095 Montpellier, France (attouch@math.univ-montp2.fr)

${ }^{\dagger}$ Partially supported by ANR-08-BLAN-0294-03.

${ }^{\ddagger}$ GREQAM UMR CNRS 6579, Université de la Méditerranée, Chateau Lafarge, Routes des Miles, 13290, Les Miles, France (antoine.soubeyran@gmail.com) 
and numerical algorithms in optimization: exploration (local search), dissimilarity (relative entropy), typology of costs to move (high, and low local costs to move), stability and robustness, adaptation.

Let us first briefly describe our dynamical decision model and then show its relationship with optimization algorithms. Solving complex problems is described as an incremental process where, step by step, the agent tries to improve his performance, getting closer and closer to a final goal. This fact has been well documented, see Lindblom [37] and the references herein. The term "agent" is taken in a broad sense, it represents either a single agent, a problem solver, or a coordinated group which acts as a single entity. We do not examine the case of interacting agents with strategic features. A performance is a way of doing, a routine that the agent is able to reproduce. Maintenance of routines requires regular training whose intensity increases with the level of performance.

Inertia and friction appear with clear evidence when the agent wants to change and improve his level of performance. Resistance to change has been recognized for a long time as a central topic in decision, economical, and social sciences, see Lewin [35], [36]. Dissimilarity is one of the main psychological and cognitive difficulties attached to change: The more dissimilar two contiguous actions are, the more difficult it is to pass from one to the other. As a general rule, improving performance requires exploration and learning, see Sobel [54]. As an original aspect of our approach, inertia and friction are described with the help of costs-to-move functions (also called costs-to-change, we use both terminologies, depending on the context), see Attouch-Soubeyran [10], [11] for a first introduction to this concept.

Let us denote by $X$ the decision space (also called, depending on the context, strategy, performance, or state space). A cost-to-move is simply a function $C: X \times$ $X \longrightarrow \mathbb{R}^{+}$. In most instances, it can be expressed with the help of a distance function (for example the square, or the square root of a distance), it may be a relative entropy, or a Bregman distance. For any $x \in X, y \in X, C(x, y)$ is the cost to move (or to change) from $x$ to $y$. In a dynamical setting, it may be useful to decompose it as $C(x, y)=t(x, y) c(x, y)$, where $t(x, y) \geq 0$ is the time spent to move from $x$ to $y$, and $c(x, y)$ is the instantaneous cost to move. Being equipped with $c: X \times X \longrightarrow \mathbb{R}^{+}$, the decision space $X$ becomes a structured space. The instantaneous cost to move $c(.,$. may be non symmetric, in order to cover dissimilarity aspects. In section 2.2 and 2.4, we examine the case where $c(.,$.$) is minorized by a distance-like function, the metric$ reflecting the difficulty for the agent to move in $X$.

The quality of the decision is measured by an instantaneous (per unit of time) gain function (also called utility, pay-off), $g: X \longrightarrow \mathbb{R} \cup\{-\infty\}$, the value $-\infty$ allowing to model in a unifying way the criteria and the constraint (we work in the maximizing context). Set $\operatorname{dom} g=\{x \in X: g(x)>-\infty\}$, the domain of $g$.

Thus, our decision model can be described with the help of the triplet

$$
\left\{\begin{array}{l}
X: \text { decision space } \\
g: X \longrightarrow \mathbb{R} \cup\{-\infty\} \text { gain function } \\
c: X \times X \longrightarrow \mathbb{R}^{+} \text {cost-to-move function. }
\end{array}\right.
$$

Let $g(y)-g(x) \geq 0$ be the instantaneous advantages to change from $x$ to $y, t(y)>0$ be the time spent to benefit of this advantage, and $\delta(x)$ be the weight the agent puts 
on his advantages to change (which depends on his character). Thus, the advantages to change from $x$ to $y$ are given by $A(x, y)=\delta(x) t(y)[g(y)-g(x)]$. To simplify this introduction take $\delta(x)=t(y)=t(x, y)=1$.

The "worthwhile-to-move principle" can be formulated as follows: At each step, the agent considers repeating his last action or changing. A transition from $x \in X$ to $y \in X$ is acceptable if the "estimated advantages to move $A(x, y)$ from $x$ to $y$ " are higher than some fraction $1 \geq \xi(x) \geq 0$ of the estimated "costs to move $C(x, y)$ from $x$ to $y$ ". This defines the worthwhile-to-move relation

$$
y \in W(x)=\{y \in X: g(y)-g(x)) \geq \xi(x) c(x, y)\} .
$$

One can associate to this relation a discrete dynamic on $X$ whose trajectories $\left(x_{n}\right)_{n \in \mathbb{N}}$ verify $x_{n+1} \in W\left(x_{n}\right)$. Following such trajectories leads to a limitation of the intermediate sacrifices to reach a final goal. When $c(x, y)$ is a distance and $\xi(x)=1$, one recovers the model described by Aubin and Ekeland in [16], called "walking on complete metric spaces".

As a general rule, the agent has limited knowledge of his environment. This may come from limited physical, time resources, see Rumelt [46], or from bounded cognitive abilities, see Simon [49], [50]. The agent has to explore and learn in order to find a satisfactory action. This requires evaluating the per unit of time payoff $g(y)$ around the present action $x \in X$. At each step, the agent explores a set $E(x, r(x))$ around $x$, where $r(x)$ is a control parameter which measures the intensity of the exploration effort, see Aubin and Lesne [17] for a model of exploration. Then he solves the qualitative inclusion

$$
y \in E(x, r(x)) \subset X .
$$

In this context, optimizing becomes an incremental process, where at each step, starting from $x_{n}$, the agent considers maximizing his net gain $g(y)-\theta_{n} c\left(x_{n}, y\right)$ (the gain which is attached to $y$ minus the cost to move from $x_{n}$ to $\left.y\right)$ over the exploration set $E\left(x_{n}, r_{n}\right)$.

When taking account of $\delta(x), t(y)$ and $t(x, y)$ as general parameters, one obtains a new class of algorithms

$$
x_{n+1} \in \epsilon_{n}-\operatorname{argmax}\left\{g(y)-\theta_{n} c\left(x_{n}, y\right): y \in E\left(x_{n}, r_{n}\right)\right\},
$$

which are called "local search proximal algorithms".

The parameters reflect various behavioral aspects of the agent

$$
\left\{\begin{array}{l}
\epsilon_{n}: \text { psychological (motivation) } \\
\theta_{n}: \text { cognitive (reactivity, speed) } \\
r_{n}: \text { exploration, learning. }
\end{array}\right.
$$

These algorithms are naturally linked with several classical optimization algorithms. Firstly, the terminology suggests the close connection with proximal algorithms. By taking $X=H$ a Hilbert space, $c(x, y)=\|x-y\|^{2}, \theta_{n}=\frac{1}{2 \lambda_{n}}>0, f:=-g, \epsilon_{n}=0$, and $E\left(x_{n}, r_{n}\right)=H$, the local search proximal algorithm reduces to the classical proximal algorithm

$$
x_{n+1} \in \operatorname{argmin}\left\{f(y)+\frac{1}{2 \lambda_{n}}\left\|y-x_{n}\right\|^{2}: \quad y \in H\right\} .
$$


We shall interpret the quadratic terms $\left\|y-x_{n}\right\|^{2}$ as low local costs to move.

An important feature of the local search proximal algorithms is the presence of the exploration set $E\left(x_{n}, r_{n}\right)$. In the classical proximal algorithm, $E\left(x_{n}, r_{n}\right)$ is the whole space, that's not too much realistic when one wants to describe real world decision processes. In this respect, local search proximal algorithms bear natural connections with trust-region methods. Local search proximal algorithms are adaptive. The agent's vision of the landscape evolves with time, as well as the agent's motivation. At a mathematical level, at each step, the parameters are updated, and our dynamics are non-autonomous.

The paper is organized as follows. In section 2, worthwhile-to-move behaviors are introduced as set-valued relationships. We study some general properties of the associated discrete-time trajectories, and pay particular attention to the interpretation of their stationary points. Then, we briefly outline a typology of costs-to-move and their connections with worthwhile-to-move dynamical properties. In section 3, we introduce optimization features in the model, and the so-called "local search proximal algorithms". Convergence results are proved successively in the high local costs-tomove case, and then in the low local costs-to-move case. In section 4, we complete this study by some stability and robustness results. In section 5 , we end the paper by making comparisons with some other optimization algorithms.

\section{The worthwhile-to-move set-valued relationship}

We present some general properties of the worthwhile-to-move set-valued relationship, and of its discrete-time trajectories. These results will be useful in the next section for studying local search proximal algorithms.

\subsection{The balance between advantages and costs-to-move}

We model "muddling through" behaviors (making small steps, improving step by step, [37]) by comparing advantages and costs to change at each step. The point is that most decisions are state-dependent, part of a dynamic decision-making process. They represent intermediate decisions to reach well defined goals.

Let us suppose that, starting from $x$, the agent has some motivation to change, and considers moving from $x \in X$ to some $y \in X$. He explores around $x \in X$, within an exploration set $E(x, r(x))$ of size $r(x) \geq 0$, to estimate and compare state-dependent intermediate advantages $A(x, y) \in \mathbb{R}$ and costs $C(x, y) \in \mathbb{R}^{+}$to move from $x$ to $y$. The size $r(x)$ of the exploration set $E(x, r(x))$ is a positive parameter which measures the intensity of the exploration process (it may be the volume of the exploration set, the duration of the exploration, the effort spent to explore). To simplify, we consider only scalar advantages and costs. They are reference-dependent, the reference being, at each step, the state $x$ of departure, then $y$, and so on. The case of multidimensional advantages and costs to move $A(x, y) \in V, C(x, y) \in V$ requires further mathematical tools, see Soubeyran and al. [55].

According to the "worthwhile-to-move" principle, an acceptable move is such that the estimated advantages are higher than some proportion $1 \geq \xi(x) \geq 0$ of the estimated 
costs:

$$
A(x, y) \geq \xi(x) C(x, y) .
$$

A move satisfies the "worthwhile-to-move" principle if and only if it satisfies this inequality. The sacrificing rate is $1-\xi(x)$, the portion of the costs to move which the agent does not put in the balance is $(1-\xi(x)) C(x, y)$. The non sacrificing rate is $\xi(x)$. The "worthwhile-to-move" set at $x \in X$ is defined by

$$
W(x)=\{y \in X: A(x, y) \geq \xi(x) C(x, y)\} \subset X .
$$

This defines a "worthwhile-to-move" set-valued relationship $x \in X \longmapsto W(x) \subset X$. We assume that state-dependent advantages and costs to move are zero if the agent stays at $x \in X: A(x, x)=C(x, x)=0$ for all $x \in X$. Thus $x \in W(x)$ for all $x \in X$. We also assume that $C(x, y)>0$ for $y \neq x$.

Let us reformulate this relationship by using a standard goal function $g: x \in X \longmapsto$ $g(x) \in \mathbb{R}$. The real number $g(x)$ represents the instantaneous utility of the agent at state $x$. Starting from a known couple $(x, g(x)) \in X \times \mathbb{R}$, the agent does not know the values $g(y)$ of his utility function, for states $y \neq x$, without exploration around $x$. Intermediate advantages to move are

$$
A(x, y)=\delta(x) t(y)(g(y)-g(x))
$$

where $t(y) \geq 0$ is the intermediate exploitation time, i.e., the length of time during which the agent hopes to benefit or choose to exploit his instantaneous advantages to move $g(y)-g(x) \geq 0$. The weight the agent puts on this advantage to change is $\delta(x)>0$. Because costs to move are non negative, a "worthwhile-to-move" choice $y \in W(x)$ improves: $y \in W(x) \Longrightarrow g(y) \geq g(x)$. Costs to move can be zero, in absence of friction. In this case of no inertia, if the exploitation time $t(y)>0$ is strictly positive, the worthwhile-to-move relation reduces to improving: $y \in W(x) \Longleftrightarrow g(y) \geq g(x)$.

The "worthwhile-to-move" principle defines an acceptable transition process $x_{n+1} \in$ $W\left(x_{n}\right), n \in \mathbb{N}$. At each step, when moving from $x_{n}$ to $x_{n+1}$, intermediate sacrifices are not too high, advantages to move $A\left(x_{n}, x_{n+1}\right)$ are greater than some fraction $\xi\left(x_{n}\right) \geq 0$ of costs to move $C\left(x_{n}, x_{n+1}\right)$. At each step, the agent improves his goal from $g\left(x_{n}\right)$ to $g\left(x_{n+1}\right) \geq g\left(x_{n}\right), n \in \mathbb{N}$. The "worthwhile-to-move" principle governs a lot of weakly goal-oriented behaviors, where, at each step, an agent, starting from the current state, tries both to improve, and to balance local advantages to move to a minimal fraction of costs-to-move.

\subsection{The "local action" property}

Let us show a general situation where an incremental "worthwhile-to-move" behavior has the "local action" property, i.e., for $n$ large enough two contiguous actions $x_{n}$ and $x_{n+1}$ are close to each other. Local action is a consequence, not an hypothesis.

Theorem 2.1. Consider a worthwhile-to-move set-valued mapping of the following form: $x \mapsto W(x) \subset X$ with

$$
W(x)=\{y \in X: g(y)-g(x) \geq c(x, y)\} \subset X .
$$


Suppose that properties (H0) and (H1) hold:

$(H 0)(X, d)$ is a metric space and there exists a function $\theta: \mathbb{R}^{+} \rightarrow \mathbb{R}^{+}$with $(\theta(r) \rightarrow 0) \Rightarrow(r \rightarrow 0)$ such that for every $x, y \in X$

$$
c(x, y) \geq \theta(d(x, y))
$$

(H1) $g($.$) is upper bounded.$

Then, for any trajectory $x_{n+1} \in W\left(x_{n}\right), n \in \mathbb{N}$ of the worthwhile-to-move inclusion, which starts from a feasible point $x_{0} \in$ domg, the following properties hold:

i) $d\left(x_{n}, x_{n+1}\right) \rightarrow 0$ as $n \rightarrow+\infty$ and $g\left(x_{n}\right) \rightarrow g^{*}<+\infty$ increasingly.

ii) $\sum_{n=0}^{+\infty} \theta\left(d\left(x_{n}, x_{n+1}\right)\right)<+\infty$.

iii) If, moreover, $c(x, y) \geq \theta_{0} d(x, y)$ for some $\theta_{0}>0$ and $(X, d)$ is a complete metric space, then the sequence $\left(x_{n}\right)_{n \in \mathbb{N}}$ converges in $(X, d)$.

Proof. : i) By definition of $W\left(x_{n}\right), x_{n+1} \in W\left(x_{n}\right)$ and assumption (H0), we have

$$
\begin{aligned}
g\left(x_{n+1}\right)-g\left(x_{n}\right) & \geq c\left(x_{n}, x_{n+1}\right) \\
& \geq \theta\left(d\left(x_{n}, x_{n+1}\right)\right) .
\end{aligned}
$$

Inequality (9) shows that the sequence of goals $\left\{g\left(x_{n}\right), n \in \mathbb{N}\right\}$ is increasing ( $(\theta($.$) is a$ nonnegative function). By assumption (H0), $g\left(\right.$.) is upper bounded, i.e., $\sup _{X} g<+\infty$. As a consequence, the sequence $\left(g\left(x_{n}\right)\right)$ is increasing and upper bounded, and hence converges to some limit $g^{*} \leq \sup _{X} g<+\infty$. It follows from (9) that $\theta\left(d\left(x_{n}, x_{n+1}\right)\right)$ tends to zero as $n$ goes to infinity. We now rely on the property of $\theta,(\theta(r) \rightarrow 0 \Rightarrow r \rightarrow 0)$, to conclude that the distance between two successive states tends to zero, $d\left(x_{n}, x_{n+1}\right) \rightarrow 0$ as $n \rightarrow+\infty$.

ii) By adding inequalities (9) from $n=0$ to $m$, using the fact that the instantaneous utility function is bounded from above, one obtains

$$
+\infty>\sup _{X} g-g\left(x_{0}\right) \geq g\left(x_{m+1}\right)-g\left(x_{0}\right) \geq \sum_{n=0}^{m} \theta\left(d\left(x_{n}, x_{n+1}\right)\right) .
$$

As a consequence, the serie $\sum_{n=0}^{+\infty} \theta\left(d\left(x_{n}, x_{n+1}\right)\right)<+\infty$ is convergent.

iii) Suppose now that $c(x, y) \geq \theta_{0} d(x, y)$ for some $\theta_{0}>0$, i.e., $\theta(r)=\theta_{0} r$. We deduce that the serie $\sum_{n=0}^{+\infty} d\left(x_{n}, x_{n+1}\right)<+\infty$. As a consequence $d\left(x_{n}, x_{m}\right) \leq$ $\sum_{k=n}^{k=m-1} d\left(x_{k}, x_{k+1}\right)$ tends to zero as $n$ and $m$ tend to infinity, which means that $\left(x_{n}\right)_{n \in \mathbb{N}}$ is a Cauchy sequence in $(X, d)$. When $(X, d)$ is a complete metric space, we finally obtain that the sequence $\left(x_{n}\right)_{n \in \mathbb{N}}$ converges in $(X, d)$.

\subsection{Behavioral rest points}

A performance $x^{*} \in X$ is said to be a rest point if, for any $y \in X$ with $y \neq x^{*}$, it is not worthwhile to move from $x^{*}$ to $y$. This is equivalent to say that $x^{*} \in X$ is a stationary point of the "worthwhile-to-move" relationship $x \in X \longmapsto W(x) \subset X, W\left(x^{*}\right)=\left\{x^{*}\right\}$. In this case, the agent has no further incentive to move. Thus, $x^{*} \in X$ is a rest point 
iff for any $y \neq x^{*}, A\left(x^{*}, y\right)<\xi\left(x^{*}\right) C\left(x^{*}, y\right)$. We say that $x^{*} \in X$ is a weak rest point iff for any $y \neq x^{*}, A\left(x^{*}, y\right) \leq \xi\left(x^{*}\right) C\left(x^{*}, y\right)$.

A performance $x^{*} \in X$ is said to be a "behavioral rest point" with respect to a given initial data $x_{0} \in X$, if it is a rest point, and starting from the given state $x_{0} \in X$, it can be reached, following a "worthwhile-to-move" (acceptable with not too many intermediate sacrifices) transition process $x_{n} \mapsto W\left(x_{n}\right), n \in \mathbb{N}$.

Ky Fan theorem, [53], gives conditions over the net incremental gain "to move instead of to stay"

$$
\Delta(x, y)=A(x, y)-\xi(x) C(x, y)
$$

which guarantee existence of a (weak) rest point.

Theorem 2.2. (Ky Fan) Let $X \subset \Upsilon$ be a non empty convex set in a topological vector space $\Upsilon$. Let $\Delta:(x, y) \in X \times X \longmapsto \Delta(x, y) \in$ be such that:

1. for each $x \in X, \Delta(x, y)$ is a quasi-concave function of $y \in X$,

2. for each $y \in X, \Delta(x, y)$ is a lower semicontinuous function of $x \in X$,

3. $\Delta(x, x) \leq 0$ for all $x \in X$,

4. $X$ is compact.

Then there exists a point $x^{*} \in X$ such that $\Delta\left(x^{*}, y\right) \leq 0$ for all $y \in X$.

But this statement does not tell us why the agent is there, and how he has reached such a rest point. Procedural rationality considers the more realistic case of a behavioral rest point. The story tells us where the agent starts from, and, step by step, which acceptable paths he is supposed to follow to reach or to be locked in a behavioral rest point. From a mathematical point of view, this corresponds to a dynamical approach to equilibrium. This can be the starting point for numerical methods allowing to find equilibria and hopefully solve optimization problems.

\subsection{Typology of costs-to-move}

Because of space limitation, and the huge variety of costs to change, we limit ourselves to the following simplified mathematical description of costs of moving :

The set of alternatives $X$ is a complete metric space. The distance $d(x, y)$ between two alternatives $x \in X$ and $y \in X$ is an index of dissimilarity between them. The physical costs of moving $C(x, y)$ from $x$ to $y$ can be decomposed into

$$
C(x, y)=e(x, y) d(x, y)=t(x, y) c(x, y) .
$$

These formula define the per unit of distance cost to move $e(x, y) \geq 0$ and the per unit of time cost to move $c(x, y) \geq 0$. We also suppose that costs to move are zero if the agent does not move: $C(x, x)=0$. Efforts per unit of distance are not symmetrical: $e(y, x) \neq e(x, y)$, so costs to move are not symmetrical either. We can have $C(x, y) \neq$ $C(y, x)$. This justifies the use of relative entropy such as Kullback-Liebler or Bregman distances. 
Regarding to the dependence of the costs-to-move with respect to the distance, we distinguish two classes:

1. "High local costs-to-move" correspond to a minimum per unit of distance effort of moving $e(x, y) \geq \underline{e}>0$.

Let us examine the important case where $C(x, y)=\underline{e} d(x, y)$, whose mechanical counterpart is dry friction, see Adly, Attouch and Cabot, [2]. Suppose $x^{*} \in X$ is a rest (stationary) point. Then, for any $y$ belonging to a neighborhood of $x^{*} \in X$

$$
g(y) \leq g\left(x^{*}\right)+\underline{e} d\left(\left(x^{*}, y\right) .\right.
$$

Returning to the cost minimization version, with $f=-g$, we recover

$$
f(y) \geq f\left(x^{*}\right)-\underline{e} d\left(\left(x^{*}, y\right),\right.
$$

which is an approximate critical point, as provided by Ekeland's $\epsilon$-variational principle (here $\epsilon=\underline{e}$ ).

This situation is examined in section 3.3.

2. "Low local costs to move" is the complementary class. This includes the important case $C(x, y)=\underline{e} d(x, y)^{2}$, whose mechanical counterpart is viscous friction.

Let us briefly describe it. Because of the presence of the square, small changes induce very small costs (like passing from 1/10 to 1/100). In that case, convergence is more difficult to prove, it requires extra geometrical assumptions on the gain or utility function $g$ (like quasi-convexity or analyticity).

Suppose $x^{*} \in X$ is a rest (stationary) point. Then, for any $y$ belonging to a neighborhood of $x^{*} \in X$

$$
g(y) \leq g\left(x^{*}\right)+\underline{e} d\left(\left(x^{*}, y\right)^{2} .\right.
$$

Returning to the cost minimization version, with $f=-g$, we recover

$$
f(y) \geq f\left(x^{*}\right)-\underline{e} d\left(\left(x^{*}, y\right)^{2},\right.
$$

which expresse that 0 is a proximal subgradient of $f$ at $x^{*}$, see [45] (Definition 8.45). When $g$ is smooth at $x^{*}$, one recovers a classical critical point.

A result illustrating this situation (local search and proximal algorithm) is given in section 3.4.

Dependence of costs to move with respect to time is a rich topic. Recall that $t(x, y)$ is the time spent to physically move from $x$ to $y$. Reactivity costs to move correspond to instantaneous costs to move $c(x, y)=\rho(x, d(x, y) / t(x, y))$ depending on the mean speed of moving $v(x, y)=d(x, y) / t(x, y)$ (Attouch and Soubeyran, [10]). In this kind of situation, costs to move increase more or less with speed.

Using entropy-like function to measure dissimilarity properties is widely used in statistics. A general mathematical modeling can be obtained by using Bregman distance: Given $X=\mathbb{R}^{n}$ and $\Phi: X \mapsto \mathbb{R} \cup+\{\infty\}$ which is convex and differentiable

$$
D_{\Phi}(x, y)=\Phi(x)-\Phi(y)-\left\langle\Phi^{\prime}(y), x-y\right\rangle
$$

is the Bregman distance associated to $\Phi$. For example, the Kullback-Leibler relative entropy can be obtained by taking $\Phi(x)=\sum x_{j} \ln \left(x_{j}\right)-x_{j}$ on the positive orthant. One may consult [18] for further examples and a recent account on the using of this notion in proximal algorithms. 


\section{Local search proximal algorithms as worthwhile-to- move optimizing behaviors}

\subsection{Clairevoyance property}

Consider that, at each step, the agent chooses the same radius of exploration $r\left(x_{n}\right)=$ $r>0, n \in \mathbb{N}$. The "local action" property, theorem 2.1 shows that, being at $x_{n}$, for $n$ large enough the next iterate $x_{n+1}$ lies inside the exploration ball of constant radius. Thus, it is reasonable to assume that, after a finite time, the agent will optimize. This is a powerful result which shows when, under inertia and frictions, an agent optimizes. This helps understanding the degree of validity of the "as if " hypothesis of economists (Friedman, [27]): even if agents do not optimize, it is "as if" agents optimize.

\subsection{Local search and proximal algorithms.}

A natural way to improve the "as if hypothesis" consists of introducing inertia costs into classical optimization programs. Optimization algorithms ignore inertia costs, except, in a very implicit way, proximal algorithms (Iusem, [30]; Attouch and Teboulle [14]; Attouch and Bolte [4]). We have to interpret the added regularization term which characterizes proximal algorithms as a "cost to change". This quite simple but important interpretation completely changes our view on the "as if hypothesis". Our model shows that agents can manage inertia "as if " they use a new algorithm which is a mixture between the two following optimization algorithms:

i) Local search algorithms: $\sup \left\{g(y): y \in E\left(x_{n}, r\left(x_{n}\right)\right)\right\}$, where $E\left(x_{n}, r\left(x_{n}\right)\right) \subset X$ is the exploration set at $x=x_{n} \in X$, of size $r\left(x_{n}\right) \geq 0$. Hill climbing and simulated annealing algorithms belong to this class of algorithms.

ii) Proximal algorithms: $\sup \left\{g(y)-\theta_{n} c\left(x_{n}, y\right): y \in X\right\}$, where $c(x, y) \geq 0$ is the regularization term which makes the goal $g(y)$ more regular. For us, the regularization term is a cost to move, which makes proximal algorithms satisfy the "worthwhile-to-change" relationship. In proximal algorithms, the criterion which is to maximize can be interpreted as a net gain function, which is a way to handle the multi-criteria problem (improve the gain function and satisfy without too much sacrificing).

The "as if" mixture consists in solving the optimization problem

$$
\sup _{y \in E\left(x_{n}, r\left(x_{n}\right)\right)}\left(g(y)-\theta_{n} c\left(x_{n}, y\right)\right)
$$

in order to pass from $x_{n}$ to $x_{n+1}$. This "worthwhile-to-change" optimization algorithm is the "Local Search and Proximal" algorithm, LSP algorithm in short. As for classical optimization, one can assume that, at stage $n$, the agent optimizes up to some approximation level $\epsilon_{n}$,

$$
x_{n+1} \in \epsilon_{n}-\operatorname{argmax}\left\{g(y)-\theta_{n} c\left(x_{n}, y\right): y \in E\left(x_{n}, r\left(x_{n}\right)\right)\right\} .
$$

We are going to study the convergence of this algorithm in the two distinctive cases, high local costs to move and then low local costs to move. 


\subsection{LSP algorithm: the "high local costs-to-move" case}

Theorem 3.1. Given $(X, d)$ a complete metric space, let $g():. x \in X \longmapsto g(x) \in$ $\mathbb{R} \cup-\{\infty\}$ be an extended real valued function (gain, utility) which satisfies $i$ ), ii):

i) $g$ is bounded from above on $X$, set $\bar{g}=\sup _{x \in X} g(x)<+\infty$;

ii) $g$ is upper semicontinuous .

Let $c: X \times X \longmapsto \mathbb{R}^{+}$with $c(x, x)=0$ be a function which satisfies iii), iv):

iii) $c$ is continuous on $X \times X$.

iv) there exists some constant $\underline{e}>0$ such that $c(x, y) \geq \underline{e} d(x, y)$ for all $x, y \in X$.

Let us give parameters $r_{n}>0$ (radius of exploration), $\theta_{n}>0$ (sacrificing index), $\epsilon_{n}>0$ (motivation index) which verify $v$ ), vi) vii):

v) $\theta_{n} \rightarrow \theta_{\infty}$ as $n \rightarrow+\infty$, with $\theta_{\infty}>0$;

vi) $r_{n} \rightarrow r_{\infty}$ as $n \rightarrow+\infty$, with $r_{\infty}>0$;

vii) $\sum \epsilon_{n}<+\infty$.

Given some initial data $x_{0} \in X$, let $\left(x_{n}\right)_{n \in \mathbb{N}}$ be a sequence defined by the local search and proximal algorithm:

$$
x_{n+1} \in \epsilon_{n}-\operatorname{argmax}\left\{g(y)-\theta_{n} c\left(y, x_{n}\right): d\left(y, x_{n}\right) \leq r_{n}\right\} .
$$

Then, the sequence $\left(x_{n}\right)_{n \in \mathbb{N}}$ converges in $X$ to some $x_{\infty}$ which satisfies the following variational property:

$$
x_{\infty} \in \operatorname{argmax}\left\{g(y)-\theta_{\infty} c\left(y, x_{\infty}\right): d\left(y, x_{\infty}\right)<r_{\infty}\right\} .
$$

Proof. a) By taking $y=x_{n}$ in the definition (13) of $x_{n+1}$, one obtains that, up to $\epsilon_{n}$, it is worthwhile to move from $x_{n}$ to $x_{n+1}$ :

$$
\epsilon_{n}+g\left(x_{n+1}\right)-g\left(x_{n}\right) \geq \theta_{n} c\left(x_{n+1}, x_{n}\right) .
$$

By $v$ ), we have $\theta_{n} \rightarrow \theta_{\infty}>0$. Hence, there exists some $\underline{\theta}>0$ such that $\theta_{n} \geq \underline{\theta}$ for all $n \in \mathbb{N}$.

Using this minorization together with $i v$ ) in (15) yields

$$
\epsilon_{n}+g\left(x_{n+1}\right)-g\left(x_{n}\right) \geq \underline{\theta} \underline{e} d\left(x_{n+1}, x_{n}\right) .
$$

Summing up these inequalities from $n=0$ to $p$, one obtains

$$
\sum_{n=0}^{p} \epsilon_{n}+g\left(x_{p+1}\right) \geq g\left(x_{0}\right)+\underline{\theta} \underline{e} \sum_{n=0}^{p} d\left(x_{n+1}, x_{n}\right) .
$$

Using $i$ ) and vii), it follows that, for all $p \in \mathbb{N}$

$$
\sum_{n=0}^{p} d\left(x_{n+1}, x_{n}\right) \leq \frac{1}{\underline{\theta} \underline{e}}\left(\sum_{n=0}^{+\infty} \epsilon_{n}+\bar{g}-g\left(x_{0}\right)\right)<+\infty .
$$

The above majorization being independent of $p$, it follows

$$
\sum_{n=0}^{+\infty} d\left(x_{n+1}, x_{n}\right)<+\infty .
$$

From (19), by using a classical argument based on the triangle inequality, one immediately obtains that $\left(x_{n}\right)_{n \in \mathbb{N}}$ is a Cauchy sequence in the complete metric space $(X, d)$ and hence converges. Set 


$$
x_{n} \rightarrow x_{\infty} \text { as } n \rightarrow \infty .
$$

b) Let us show that

$$
x_{\infty} \in \operatorname{argmax}\left\{g(y)-\theta_{\infty} c\left(y, x_{\infty}\right): d\left(y, x_{\infty}\right)<r_{\infty}\right\} .
$$

To that end, let us fix some arbitrary $y \in X$ such that $d\left(y, x_{\infty}\right)<r_{\infty}$. Since $x_{n} \rightarrow x_{\infty}$ and $r_{n} \rightarrow r_{\infty}>0$ as $n \rightarrow+\infty$, for $n$ sufficiently large, $d\left(y, x_{n}\right)<r_{n}$.

Thus, by definition (13) of $x_{n+1}$

$$
\epsilon_{n}+g\left(x_{n+1}\right)-\theta_{n} c\left(x_{n+1}, x_{n}\right) \geq g(y)-\theta_{n} c\left(y, x_{n}\right) .
$$

Using $c\left(x_{n+1}, x_{n}\right) \geq 0$ and $\theta_{n}>0$, we deduce

$$
\epsilon_{n}+g\left(x_{n+1}\right) \geq g(y)-\theta_{n} c\left(y, x_{n}\right) .
$$

By using the upper-semicontinuity of $g$, the continuity of $c$, together with $\epsilon_{n} \rightarrow 0$ and $\theta_{n} \rightarrow \theta_{\infty}>0$, we derive from the above inequality

$$
\begin{aligned}
g\left(x_{\infty}\right) & \geq \limsup _{n} g\left(x_{n+1}\right) \\
& \geq g(y)-\theta_{\infty} c\left(y, x_{\infty}\right),
\end{aligned}
$$

which ends the proof.

Remark 3.2. Theorem 3.1 provides a dynamical proof of Ekeland's $\epsilon$-variational principle. By taking $c(x, y)=d(x, y)$ and the whole space as the exploration set, one obtains the existence of some $x^{*} \in X$ such that

$$
x^{*} \in \operatorname{argmax}\left\{g(y)-\theta d\left(y, x^{*}\right): y \in X\right\} .
$$

Moreover, as a straight consequence of (18), by setting $\epsilon=\sum_{n=0}^{+\infty} \epsilon_{n}$ one obtains

$$
d\left(x^{*}, x_{0}\right) \leq \frac{1}{\theta}\left(\epsilon+\bar{g}-g\left(x_{0}\right)\right)<+\infty,
$$

which completes the proof of Ekeland's theorem. The link between Ekeland's theorem and dissipative dynamical systems has been first recognized in Aubin and Ekeland [16], Ch. 5, and further in Attouch and Soubeyran [11]. The novelty of our approach lies in the cognitive interpretation and the local features of the discrete dynamic (algorithm) whose trajectories converge to a solution of Ekeland's theorem.

Remark 3.3. In theorem 3.1, the local search and proximal algorithm considers any given sequence $\left\{r_{n}>0 ; n \in \mathbb{N}\right\}$ of size of exploration sets which converges to a positive limit $r_{\infty}>0$. Thus, it gives a partial answer to the recursive costs problem which is "how to choose, each step, the size $r\left(x_{n}\right) \geq 0$ of the exploration set" i.e., "how much to explore, depending on the costs of exploration"? This helps to pave the way to solve the famous "effort-accuracy" problem which balances exploration costs and the quality of the decision, see Payne and alii, [43]. For a survey, one can consult the "decision field theory" of Busemeyer-Diederich, [21]. Proximal algorithms take, each step $x_{n} \in X$, the same exploration set, the whole space $E\left(x_{n}, r\left(x_{n}\right)\right)=X$. From a behavioral point of view this is not reasonable, because this means to solve, each step, a global optimization problem (the substantive case)! 


\subsection{LSP algorithm: the "low local costs-to-move" case}

In the following result, we prove convergence of the LSP algorithm in the "low local costs-to-move" case. Because of the low dissipation property of the dynamic, we need to make geometrical assumptions on the gain function $g$. We consider here the case of a quasi-concave gain function, which is an interesting situation in economics. The following argument is much in the lines of the recent paper of Goudou-Munier, [28] on gradient methods for quasi-convex functions.

Theorem 3.4. Let $X$ be a real Hilbert space. Let $C \subset X$ be a closed convex nonempty subset of $X$ (set of constraints, resources). Let $g():. x \in X \longmapsto g(x) \in \mathbb{R}$ be a function (gain, utility) which satisfies i), ii) and iii):

i) $g$ is upper bounded on $C$, let $\bar{g}<+\infty$ be the supremum of $g$ on $C$.

ii) $g$ is a smooth function (continuously differentiable).

iii) $g$ is quasi-concave (with convex upper level sets).

Given some initial data $x_{0} \in X$, let $\left(x_{n}\right)_{n \in \mathbb{N}}$ be a sequence defined by the local search and proximal algorithm with clairvoyance radius $r>0$ and parameter $\theta_{n}>0$ :

$$
x_{n+1} \in \operatorname{argmax}\left\{g(y)-\theta_{n}\left\|x_{n}-y\right\|^{2}: y \in C,\left\|y-x_{n}\right\| \leq r\right\},
$$

where $\left(\theta_{n}\right)$ is a sequence of positive numbers which is bounded from above and bounded from below by a positive number, say

$$
0<\underline{\theta} \leq \theta_{n} \leq \bar{\theta}<+\infty \quad \forall n \in \mathbb{N} .
$$

Then, the sequence $\left(x_{n}\right)_{n \in \mathbb{N}}$ weakly converges in $X$ to some $x_{\infty}$. When $X$ is a finite dimensional space, this limit can be characterized as a critical point of $g$ over $C$, namely

$$
-\nabla g\left(x_{\infty}\right)+N_{C}\left(x_{\infty}\right) \ni 0
$$

where $N_{C}\left(x_{\infty}\right)$ is the (outward) normal cone to $C$ at $x_{\infty}$.

Proof. By taking $y=x_{n}$ in (24), we obtain

$$
g\left(x_{n+1}\right)-g\left(x_{n}\right) \geq \theta_{n}\left\|x_{n+1}-x_{n}\right\|^{2},
$$

which expresses that it is worthwhile to move from $x_{n}$ to $x_{n+1}$. Summing up these inequalities, using $i$ ) and $0<\underline{\theta} \leq \theta_{n}$ yields

$$
\sum_{n=0}^{\infty}\left\|x_{n+1}-x_{n}\right\|^{2} \leq \frac{1}{\underline{\theta}}\left(\bar{g}-g\left(x_{0}\right)\right)<+\infty .
$$

As a consequence,

$$
\left\|x_{n+1}-x_{n}\right\| \rightarrow 0 \text { as } n \rightarrow+\infty .
$$

Hence, for $n$ large enough, $\left\|x_{n+1}-x_{n}\right\|<r$, which implies that the supremum in (24) is actually achieved at $x_{n+1}$ which belongs to the interior of the ball $\mathbb{B}\left(x_{n}, r\right)$ with center $x_{n}$ and radius $r>0$. Thus, when writing first-order optimality conditions, for $n$ large enough, the exploration constraint is not active, and one obtains

$$
-\nabla g\left(x_{n+1}\right)+N_{C}\left(x_{n+1}\right)+2 \theta_{n}\left(x_{n+1}-x_{n}\right) \ni 0 .
$$

Convergence of the sequence of values $\left(g\left(x_{n}\right)\right)_{n \in \mathbb{N}}$ is an immediate consequence of (26). Indeed, it is an increasing upper-bounded sequence. Set 


$$
g_{\infty}=\lim _{n \rightarrow+\infty} g\left(x_{n}\right) .
$$

In order to prove convergence of the sequence $\left(x_{n}\right)_{n \in \mathbb{N}}$ it will be useful to introduce the set

$$
S=\left\{x \in C: g(x) \geq g_{\infty}\right\}
$$

and prove (in the lines of Opial lemma) that:

a) For every $a \in S, \lim _{n \rightarrow+\infty}\left\|x_{n}-a\right\|^{2}$ exists.

b) Every weak limit point of the sequence $\left(x_{n}\right)_{n \in \mathbb{N}}$ belongs to $\mathrm{S}$.

Indeed, by convexity of the norm

$$
\left\|x_{n}-a\right\|^{2}-\left\|x_{n+1}-a\right\|^{2} \geq 2\left\langle x_{n+1}-a, x_{n}-x_{n+1}\right\rangle .
$$

By (29) there exists some $\xi_{n} \in N_{C}\left(x_{n+1}\right)$ such that

$$
x_{n}-x_{n+1}=\frac{1}{2 \theta_{n}}\left(-\nabla g\left(x_{n+1}\right)+\xi_{n}\right) .
$$

Combining (30) and (31)

$$
\left\|x_{n}-a\right\|^{2}-\left\|x_{n+1}-a\right\|^{2} \geq \frac{1}{\theta_{n}}\left\langle x_{n+1}-a,-\nabla g\left(x_{n+1}\right)+\xi_{n}\right\rangle .
$$

We use the quasi-concavity assumption on $g$ in order to prove that

$$
\left\langle x_{n+1}-a,-\nabla g\left(x_{n+1}\right)+\xi_{n}\right\rangle \geq 0 .
$$

To that end we consider the set

$$
D_{n}=\left\{x \in C: g(x) \geq g\left(x_{n+1}\right)\right\}
$$

which is an upper level set over $C$ of the function $g$. Because of the quasi-concavity of $g$ and of the convexity of $C$ this a closed convex subset of $X$. By a classical geometrical argument (Rockafellar and Wets, [45], ch. 10)

$$
N_{D_{n}}\left(x_{n+1}\right)=-\nabla g\left(x_{n+1}\right)+N_{C}\left(x_{n+1}\right) .
$$

Hence,

$$
-\nabla g\left(x_{n+1}\right)+\xi_{n} \in N_{D_{n}}\left(x_{n+1}\right)
$$

and as $a \in S \subset D_{n}$ (recall that $g(a) \geq g\left(x_{n+1}\right)$ ) we obtain (33). Returning to (32) we obtain that $\left\|x_{n}-a\right\|^{2}$ is a decreasing sequence, hence converges, which proves point a). Concerning point b), recall that $g_{\infty}=\lim _{n \rightarrow+\infty} g\left(x_{n}\right)$. As $g$ is concave continuous, it is upper semicontinuous for the weak topology of $X$. Hence any weak limit point $x^{*}$ of the sequence $\left(x_{n}\right)_{n \in \mathbb{N}}$ satisfies $g\left(x^{*}\right) \geq \lim g\left(x_{n}\right)=g_{\infty}$. As $C$ is a closed set and $x_{n} \in C$ for all $n \in \mathbb{N}$ we still have that $x^{*} \in C$ at the limit. These two results imply $x^{*} \in S$, which is point $b$ ).

The Opial argument allows us to deduce that the whole sequence $\left(x_{n}\right)_{n \in \mathbb{N}}$ weakly converges. This sequence is bounded because $\lim _{n \rightarrow+\infty}\left\|x_{n}-a\right\|^{2}$ exists for every $a \in S$ and $S \neq \emptyset$. Suppose that the sequence $\left(x_{n}\right)_{n \in \mathbb{N}}$ has two weak limit points, set

$$
x_{n_{1}} \rightarrow x_{1}^{*} \text { and } x_{n_{2}} \rightarrow x_{2}^{*} .
$$


By point b), $x_{1}^{*}$ and $x_{2}^{*}$ belong to $S$.

Using point a), $\lim _{n \rightarrow+\infty}\left\|x_{n}-x_{1}^{*}\right\|^{2}$ and $\lim _{n \rightarrow+\infty}\left\|x_{n}-x_{2}^{*}\right\|^{2}$ exist. Subsequently,

$$
\lim _{n \rightarrow+\infty}\left(\left\|x_{n}-x_{1}^{*}\right\|^{2}-\left\|x_{n}-x_{2}^{*}\right\|^{2}\right) \text { exists }
$$

which after simplification yields

$$
\lim _{n \rightarrow+\infty}\left\langle x_{n}, x_{2}^{*}-x_{1}^{*}\right\rangle \text { exists. }
$$

Specializing this result to the two subsequences $x_{n_{1}}$ and $x_{n_{2}}$ which weakly converge respectively to $x_{1}^{*}$ and $x_{2}^{*}$,

$$
\left\langle x_{1}^{*}, x_{2}^{*}-x_{1}^{*}\right\rangle=\left\langle x_{2}^{*}, x_{2}^{*}-x_{1}^{*}\right\rangle
$$

that is, $\left\|x_{1}^{*}-x_{2}^{*}\right\|^{2}=0$. Thus the sequence $\left(x_{n}\right)_{n \in \mathbb{N}}$ has a unique weak limit point, and hence weakly converges in $X$ to some $x_{\infty}$.

Suppose now that $X$ is a finite dimensional Hilbert space. By passing to the limit on (29)

$$
-\nabla g\left(x_{n+1}\right)+N_{C}\left(x_{n+1}\right)+2 \theta_{n}\left(x_{n+1}-x_{n}\right) \ni 0
$$

and by using (28) together with the smoothness of $g$ (assumption ii)), the boundedness of $\left(\theta_{n}\right)$ and the closedness property of the graph of the normal cone mapping $x \mapsto$ $N_{C}(x)$, we finally obtain

$$
-\nabla g\left(x_{\infty}\right)+N_{C}\left(x_{\infty}\right) \ni 0
$$

which expresses that $x_{\infty}$ is a critical point of $g$ over $C$.

\section{$4 \quad$ Stability and robustness results}

Local search proximal algorithms inherit some of the remarkable stability and robustness properties of proximal algorithms. Just to illustrate it, let us consider the following result which concerns the "low local costs-to-move" case. Assuming the gain function $g$ to be concave, it is claimed that, after a finite number of steps, trajectories enjoy a contraction continuity property with respect to the data.

Theorem 4.1. Let $H$ be a real Hilbert space. Let $g():. x \in H \mapsto g(x) \in \mathbb{R} \cup\{-\infty\}$ be a extended real valued function which is assumed to be concave, upper semicontinuous, and proper. Let $\left(x_{n}\right)_{n \in \mathbb{N}}$ and $\left(y_{n}\right)_{n \in \mathbb{N}}$ be two sequences defined by the local search and proximal algorithm with clairvoyance radius $r>0$ and parameter $\theta_{n}>0$ :

$$
\begin{aligned}
& x_{n+1}=\operatorname{argmax}\left\{g(\xi)-\theta_{n}\left\|\xi-x_{n}\right\|^{2}: \xi \in H,\left\|\xi-x_{n}\right\| \leq r\right\} ; \\
& y_{n+1}=\operatorname{argmax}\left\{g(\xi)-\theta_{n}\left\|\xi-y_{n}\right\|^{2}: \xi \in H,\left\|\xi-y_{n}\right\| \leq r\right\},
\end{aligned}
$$

where $\left(\theta_{n}\right)$ is a sequence of positive numbers which is bounded from above and bounded from below by a positive number, say

$$
0<\underline{\theta} \leq \theta_{n} \leq \bar{\theta}<+\infty \quad \forall n \in \mathbb{N} .
$$

Then, there exists $n_{0} \in \mathbb{N}$ such that the sequence $\left(\left\|x_{n}-y_{n}\right\|\right)_{n \geq n_{0}}$ decreases with $n$. Denoting by $x_{\infty}$ and $x_{\infty}$ the respective limits of $\left(x_{n}\right)_{n \in \mathbb{N}}$ and $\left(y_{n}\right)_{n \in \mathbb{N}}$, for $n \geq n_{0}$

$$
\left\|x_{\infty}-y_{\infty}\right\| \leq\left\|x_{n}-y_{n}\right\| \leq \ldots \leq\left\|x_{n_{0}}-y_{n_{0}}\right\| .
$$


Proof. First notice that (41) can be equivalently written as the minimization problem, with $f=-g$,

$$
\inf \left\{f(\xi)+\theta_{n}\left\|\xi-x_{n}\right\|^{2}: \xi \in H,\left\|\xi-x_{n}\right\| \leq r\right\},
$$

which consists in the minimization of a strongly convex, lower semicontinuous function on a Hilbert space, and hence has a unique minimum point, called $x_{n+1}$. The same holds concerning (42) and $y_{n+1}$. Following (29), for $n$ large enough, let us say $n \geq n_{0}$ the exploration constraint is not active, and the following optimality properties hold

$$
\begin{aligned}
& -\partial g\left(x_{n+1}\right)+2 \theta_{n}\left(x_{n+1}-x_{n}\right) \ni 0 \\
& -\partial g\left(y_{n+1}\right)+2 \theta_{n}\left(y_{n+1}-y_{n}\right) \ni 0 .
\end{aligned}
$$

Let us write the monotonicity property of $-\partial g$ at $x_{n+1}$ and $y_{n+1}$, and use (46), (47) to obtain

$$
\left\langle\left(x_{n+1}-x_{n}\right)-\left(y_{n+1}-y_{n}\right), x_{n+1}-y_{n+1}\right\rangle \leq 0 \text {. }
$$

Equivalently

$$
\left\|x_{n+1}-y_{n+1}\right\|^{2} \leq\left\langle x_{n}-y_{n}, x_{n+1}-y_{n+1}\right\rangle .
$$

By using Cauchy-Schwarz inequality, we deduce that, for $n \geq n_{0}$

$$
\left\|x_{n+1}-y_{n+1}\right\| \leq\left\|x_{n}-y_{n}\right\|,
$$

which expresses that the sequence $\left(\left\|x_{n}-y_{n}\right\|\right)_{n \geq n_{0}}$ decreases with $n$.

Using the weak lower semicontinuity of the norm, and the weak convergence property of sequences $\left(x_{n}\right)$ and $\left(y_{n}\right)$ (theorem 3.4) we finally obtain

$$
\left\|x_{\infty}-y_{\infty}\right\| \leq\left\|x_{n}-y_{n}\right\| \leq \ldots \leq\left\|x_{n_{0}}-y_{n_{0}}\right\| .
$$

\section{Comparisons between local proximal, optimizing be- haviors, and optimization algorithms}

Local search proximal algorithms have been primarily designed in order to describe real life human behaviors. They can handle both complex (infinite) state space, radical uncertainty aspects and some of the physiological, psychological and cognitive limitations of the agents. They also involve optimization aspects which make them naturally linked with numerical optimization algorithms. Let us examine these different aspects, and first show the main advantages of local search proximal algorithms when the point is modeling of human optimizing behaviors.

\subsection{Comparisons between local proximal and optimizing behaviors}

The basic question is: do these algorithms provide a realistic description of the dynamical and stationary aspects of real life human behaviors? In this general context, the word algorithm is misleading since these algorithms do not pretend to numerically solve optimization problems! On the opposite, because of inertia and frictions which 
generate costs to change during transitions, they help understanding how humans can ultimately reach rest points (permanent routines) which correspond to inefficient outcomes, far from the optimum. Our algorithm can be better viewed as a discrete in time dynamical system describing human decision processes when inertia matters and changing has a cost. These algorithms involve the three basic blocks: exploration, transition with inertia, and goal-setting blocks with the corresponding control parameters. They allow to describe a large spectrum of behaviors, from muddling through behaviors, satisficing, satisficing and "worthwhile-to-move", to global optimization. In a second paper [13] we give several applications to decision sciences of the theory of "worthwhileto-move" behaviors and of the "local search proximal" algorithms. We show how the agent can overcome inertia by using adaptive behaviors involving long term goals and short term intermediate goals. Transitions are important, realistic decision-making models must take care of the well being of the agents during transitions: because of inertia, agents reject transitions with too many intermediate sacrifices (costs to change and costs to learn how to do a new action). Most classical models in decision sciences rely on global optimization. By contrast, in a human context, our approach offers much more realistic aspects, let us make out a list.

1. The state space can be infinite, and even non compact, which allows to model complex aspects.

2. The agent can have a (semicontinuous) non differentiable long term objective.

3. The exploration process takes account of the fact that the agent does not know ex ante the whole space, nor the geometry of the state space.

4. The agent has not only a long term objective, but also a short term objective. It permits him to avoid too much temporary sacrifices during the transition (because of costs to change): during transition the agent must survive!

5. The agent can have a more goal oriented objective than to improve (a "muddling through" behavior). He can set intermediate objectives, like temporary satisficing ("improving enough"). This allows to model the celebrated "intermediate goals setting process" of Vroom [57], and the famous "hard goal effect" of Locke and Latham [39].

6. The context has a conservative aspect. It includes inertia, as costs to change, like attention costs, exploration costs, learning costs, switching costs, adaptation costs.

7. The context has a dual innovative aspect. It considers learning costs, as costs to know how a do a new action (a major case of inertia). Besides "one-line" learning which can be very costly (to be obliged to do "to be able" to know), there is "off-line" learning which can be far less costly (by thinking, simulations, estimations, without being obliged to do a new action).

8. The exploration process is not necessary local, the agent is not obliged to do small steps. The exploration process can be semi local, visiting neighbours of neighbours, neighbours of neighbours of neighbours. Indeed, the convergence result shows that an agent can start doing big steps, but will end doing small steps, in an endogenous way.

9. The approach can help to escape from local maxima (minima). The agent is allowed to do big and decreasing steps at the very beginning, and even from time to time along a subsequence.

10. The exploration process is adaptive, linking the temporary satisficing process 
to the amount of exploration which must be done at each step. Hence it can be an economizing heuristic, escaping to explore too much or to few.

11. The decision-making process is quite realistic (in a human, real life context). It does not require that the agent tosses a coin to decide to do someting or not. The agent is not supposed to be an engineer who can use sophisticated mathematical tools (exponentials, approximating a limit distribution in a finite number of steps,...).

\subsection{Comparison with proximal algorithms}

Our algorithms are closely connected to proximal algorithms. The main difference is the presence of an exploration process, and the general form of the costs-to-move terms. In recent years, proximal algorithms have been an active field of research, with some important new results. Let us list some of them, keeping in mind that, with a reasonable effort, one can presumably transpose them to our algorithms.

1. At each step, when using (local search) proximal algorithms, one has to (approximately) solve an optimization problem (by using a numerical optimization algorithm). It may look strange to replace a single optimization problem by a sequence of optimization problems. Indeed, this procedure has many advantages. In classical proximal algorithms the quadratic cost-to-change term is usually interpreted as a regularization term. When writing first order optimality condition for

$$
x_{n+1} \in \operatorname{argmax}\left\{\phi(y)-\theta_{n}\left\|x_{n}-y\right\|^{2}: y \in H\right\},
$$

denoting $\lambda_{n}=\frac{1}{2 \theta_{n}}$ one obtains

$$
\frac{1}{\lambda_{n}}\left(x_{n+1}-x_{n}\right)-\partial \phi\left(x_{n+1}\right) \ni 0 .
$$

This equation can be interpreted as an implicit discretization of the continuous first order gradient system

$$
\dot{x}(t)-\partial \phi(x(t)) \ni 0
$$

which is called the steepest ascent method. This continous dynamical system plays a central role in optimization, differential geometry, physics and many other domains. Proximal algorithms share most of the large time convergence properties of this dynamical system. Convergence properties have been first established in the case of a concave upper semicontinous function $\phi$, see Rockafellar [44]. More recently, several studies have been devoted to the study of the non convex case. In Attouch and Bolte [4], the approach is based on the Kurdyka-Lojasiewicz inequality, which is valid for a large class of possibly non-smooth functions including real analytic or semialgebraic functions. In Hare and Sagastizabal [29], and Iusem, Pennanen and Svaiter [31], the approach is based on a local control of the defect of convexity. Kaplan and Tichatschke [33] consider proximal point approach to general variational inequality problems. One should notice that these convergence properties hold even if the initial optimization problem is ill posed with a continuum of solutions. The algorithm asymptotically selects a particular one (which depends on the initial data). 
2. Proximal dynamics still make sense in spaces without differentiable structures (just replace the square of the norm by some metric). It allows to define steepest ascent dynamics in metric spaces, for example gradient flows in the space of probability measures with applications to the Monge-Kantorovich optimal transport problem. Replacing the euclidian norm square by a Bregman distance or a relative entropy has been first motivated by the study of interior point methods in optimization, see Bauschke, Combettes and Noll [18], Eckstein [24], Iusem [30], Teboulle [56].

3. Under quite general assumptions, alternating proximal minimization algorithms allow to obtain decomposition or splitting results for structured optimization problems, see Attouch, Bolte, Redont and Soubeyran [5], [9]. In [7] Attouch, Briceno-Arias, and Combettes develop a parallel splitting method for coupled monotone inclusions, which involve proximal steps with respect to the non-smooth operators. As a striking result, the introduction of costs-to-move, and hence of proximal dynamics, has permitted to obtain, via Kurdyka-Lojasiewicz inequality, general decomposition results for nonsmooth, non-convex structured optimization problems, see Attouch, Bolte, Redont and Soubeyran [6]. Among others, these results have interesting applications to best reply dynamics for potential games, and provide new insight into the Nash equilibration problem.

\subsection{Comparison with local search algorithms}

Exploration is a basic block of our model. In this paper, we have been mostly concerned with the costs-to-move aspects. The exploration process has been first considered from a qualitative point of view. In section 3 , we have presented a mathematical study in a simplified situation, (but already significant) when the radius of exploration is constant (or, more generally, minorized by some positive number). Clearly, describing adaptive exploration strategies is a question of importance which requires further studies. These questions are central in local search algorithms. We just outline below, very briefly, some connections between local search algorithms and our study.

1. Trust-region methods are closely related to proximal algorithms, and even more to our local search proximal algorithms. This fact has been recognized by Conn, Gould and Toint in a well documented monograph [23], Notes and references to section 6.1, page 120-121. One can also consult chapter 4 of Nocedal and Wright [42] for an introduction to this subject. Recent developments of proximal algorithms for non convex problems should make even closer these two domains of research. A basic feature of trust-region methods is the construction, at each step, of a model of the gain function $g$. This is an aspect which fits well with decision modeling theory (landscape theory). It means that the agent constructs by extrapolation from the local data he has (usually an approximation of the gradient and Hessian of the gain function, at the current point), a more global representation of his environment (usually a quadratic representation). In trust-region methods, a central question is the choice of the size of the region in which the agent can trust the model. Combining these ideas with local search proximal algorithms, would lead to study adaptive algorithms of the following type

$$
x_{n+1} \in \epsilon_{n}-\operatorname{argmax}\left\{g_{n}(y)-\theta_{n} c\left(x_{n}, y\right): y \in E\left[x_{n}, r\left(x_{n}\right)\right]\right\},
$$


with $g_{n}$ and $E\left[x_{n}, r\left(x_{n}\right)\right]$ governed by some interdependent dynamical process. In [3], P. Apkarian, D. Noll and O. Prot have developed such program to minimize nonsmooth and nonconvex semi-infinite maximum eigenvalue functions.

2. To say that our algorithm is better or worse than some other existing algorithm depends on the context. Let us briefly compare it (in a human context, it is a dynamic heuristic) with the simulated annealing algorithm, which is a widely used local search optimizing algorithm (a computer context). An extensive comparison is available in [12]. The goal of a simulated annealing process is to reach (approximatively) an optimum of an unknown function on a known and finite state space, after a finite number of steps. It uses (before any local exploration) a given neighbour structure of search, a given probabilistic generation rule for new actions and a probabilistic acceptance rule for an improving or "from time to time" decreasing action. By contrast, in our model the agent does not know the content of any exploration set before doing exploration! Thus, he cannot determine ex ante the probability to pick a neighbour action, which, in this context, forbids using a probabilistic process (like in many global optimization algorithms).

\section{References}

[1] E. Aarts and K. Lenstra, Local search, Princeton University Press, 2003.

[2] S. Adly, H. Attouch and A. Cabot, Finite time stabilization of nonlinear oscillators subject to dry friction, Nonsmooth Mechanics and Analysis, Advances in Mechanics and Mathematics, 12, edited by P. Alart, O. Maisonneuve and R.T. Rockafellar, Springer, 2006.

[3] P. Apkarian, D. Noll and O. Prot, A proximity control algorithm to minimize nonsmooth and nonconvex semi-infinite maximum eigenvalue functions J. of Convex Analysis, 16, (2009), pp. 641 - 666.

[4] H. Attouch and J. Bolte, On the convergence of the proximal algorithm for non smooth functions involving analytic features, Math. Program., 116 (2009), no. 1-2, Ser. B, pp. 5-16.

[5] H. Attouch, J. Bolte, P. Redont, and A. Soubeyran, Alternating proximal algorithms for weakly coupled convex minimization problems. Applications to dynamical games and PDE's, J. of Convex Analysis, 15 (2008), pp. 485-50.

[6] H. Attouch, J. Bolte, P. Redont, and A. Soubeyran, Proximal alternating minimization and projection methods for nonconvex problems. An approach based on the Kurdyka-Lojasiewicz inequality, arXiv:0801.1780, (2008), to appear in Mathematics of Operations Research.

[7] H. Attouch, L. M. Briceno-Arias, and P. L. Combettes, A parallel splitting method for coupled monotone inclusions, SIAM J. Control Optim. 48 (2010), no. 5, pp. 3246-3270. 
[8] H. Attouch, X. Goudou, and P. Redont The heavy ball with friction method, Communication in Contemporary Mathematics, 2 (2000), pp. 1-34.

[9] H. Attouch, P. Redont, and A. Soubeyran, A new class of alternating proximal minimization algorithms with costs-to-move, SIAM J. on Optimization, 18 (2007), pp. 1061-1081.

[10] H. Attouch and A. Soubeyran, Inertia and reactivity in decision making as cognitive variational inequalities, J. of Convex Analysis, 13 (2006), pp. 207-224.

[11] H. Attouch and A. Soubeyran, A cognitive approach of the Ekeland theorem, section 3.4. of "Variational Analysis in Sobolev and BV Spaces: Applications to PDE and Optimization", Editors H. Attouch - G. Buttazzo - G. Michaille, MPS-SIAM series on Optimization, MP06, (2006).

[12] H. Attouch and A. Soubeyran, Comparison between "local proximal" and "simulated annealing" algorithms, working paper, GREQAM, (2008).

[13] H. Attouch and A. Soubeyran, From muddling through, to satisficing, to optimization: worthwhile to change adaptive behaviors, working paper, GREQAM, (2009).

[14] H. Attouch and M. Teboulle, A regularized Lotka-Volterra dynamical system as a continuous proximal-like method in optimization, J. Optim. Theory Appl., 121 (2004), pp. 541-580.

[15] J. P. Aubin, Evolution tychastique, stochastique et contingente, mimeo, 2005.

[16] J. P. Aubin and I. Ekeland, Applied non linear analysis, Wiley, 1983.

[17] J. P. Aubin and A. Lesne, Constructing and exploring wells of energy landscapes, J. Math. Phys., 46 (2005), pp. 1-16.

[18] H. H. Bauschke, P. L. Combettes and D. Noll, Joint minimization with alternating Bregman proximity operators, Pacific Journal of Optimization, 2 (2006), pp. 401424.

[19] H. Brézis, Opérateurs maximaux monotones et semi-groupes de contractions dans les espaces de Hilbert, North-Holland/Elsevier, New-York, 1973.

[20] R. E. Bruck, Asymptotic convergence of nonlinear contraction semigroups in Hilbert spaces, J. Funct. Anal., 18 (1975), pp. 15-26.

[21] J. R. Busemeyer, and A. Diederich, Survey of decision field theory, Math. Soc. Sci., 43 (2002), pp. 345-370.

[22] J. Conlisk, Why bounded rationality?, Journal of Economic Literature, 34 (1996), pp. 669-700.

[23] A. Conn, N. Gould, and P. Toint, Trust-region methods, MPS-SIAM Series on Optimization, 1996. 
[24] J. Eckstein, Nonlinear proximal point algorithms using Bregman functions with applications to convex programming, Mathematics of Operations Research, 18 (1993), pp. 202-226.

[25] I. Ekeland, On the variational principle, J. Math. Analysis Applications, 47 (1974), pp. 325-353.

[26] K. Fan, A Minimax inequality and applications, Inequalities, 3, Sisha Ed., Academic Press, New York, pp. 103-113.

[27] J. Friedman, The methodology of positive economics, essays in positive economics, University Press of Chicago, 1953.

[28] X. Goudou, and J. Munier, The gradient and heavy ball with friction dynamical systems: the quasiconvex case, Math. Program., 116 (2009), no. 1-2, Ser. B, pp. 173-191.

[29] W. Hare and C. Sagastizabal, Computing proximal points of nonconvex functions, Math. Program. Ser. B, 116 (2009), pp. 221-258.

[30] A. N. Iusem, On the dual convergence and the rate of primal convergence of Bregman's convex programming method, SIAM J. on Optimization, 1 (1991), pp. 401423.

[31] A. N. Iusem, T. Pennanen, and B. Svaiter, Inexact variants of the proximal point algorithm without monotonicity, SIAM J. on Optimization, 13 (2003), pp. 10801097.

[32] A. N. Iusem, B. F. Svaiter and J. X. Da Cruz Neto, Central paths, generalized proximal point methods, SIAM J. Control Optim., 37 (1989), pp. 566-588.

[33] A. Kaplan and R. Tichatschke, Proximal point approach and approximation of variational inequalities, SIAM J. Control Optim., 39 (2000), pp. 1136-1159.

[34] P. Klemperer, Competition when consumers have switching costs, Review of Economic Studies, 62 (1995), pp. 515-539.

[35] K. Lewin, Frontiers in group dynamics, Human Relations, 1 (1947), pp. 143-153.

[36] K. Lewin, Field theory in social science, New York, Harper and Row, 1951.

[37] C. Lindblom, The science of muddling through, Public Administrative Review, 19 (1959), pp. 79-99.

[38] B. Lipman, and R. Wang, Switching costs in frequently repeated games, Journal of Economic Theory, 93 (2000), pp. 149-190.

[39] E. Locke, G. and Latham, A theory of goal setting and task performance, Englewoods Cliffs, NJ: Prentice Hall, 1990.

[40] J. E. Martinez-Legaz and A. Soubeyran, Learning from errors, UAB, Working paper LEA, University of Barcelona, 2002. 
[41] J. E. Martinez-Legaz and A. Soubeyran, A tabu search scheme for abstract problems, J. of Math. Analysis and Applications, 73 (2007), pp. 1-8.

[42] J. Nocedal and S. Wright, Numerical Optimization, Springer Series in Operations research, 1999.

[43] J. Payne, J. Bettman, J., and E. Johnson, Adaptive strategy selection in decision making, J. of Experimental Psychology, 14 (1988), pp. 534-553.

[44] R. T. Rockafellar, Monotone operators and the proximal point algorithm, SIAM J. Control Opt, 14 (1976), pp. 877-898.

[45] R. T. Rockafellar and R. J.-B. Wets, Variational Analysis, Springer- Verlag, 2004.

[46] R. Rumelt, Inertia and transformation, mimeo, (1990).

[47] R. Selten, Features of experimentally observed bounded rationality, European Economic Review, (1998) 42, pp. 413-436.

[48] R. Selten, Aspiration adaptation theory, Journal of Mathematical Psychology, (1998) 42, pp. 191-214.

[49] H. Simon, A behavioral model of rational choice, Quaterly Journal of Economics, 69 (1955), pp. 99-118.

[50] H. Simon, From substantive to procedural rationality, Methods and Appraisal in Economics, Latsis, editor, (1967).

[51] H. Simon, The science of the artificial, 2nd Edition, Cambridge, MA: MIT Press, 1982.

[52] H. Simon, Satisficing, the New Palgrave: A dictionary of Economics, Eds Eatwell J., Milgate M., Newman P., London, Mac Millan, (1987), pp. 243-245.

[53] S. Singh, B. Watson, and P. Srivastava, Fixed point theory and best approximation: the KKM map principle, Kluwer,(1997).

[54] J. Sobel, Economists' models of learning, J. Econom. Theory, 94 (2000), pp. 241261.

[55] A. Soubeyran and B. Soubeyran, A value creation-value appropriation theory of organizational change: A variational approach, GREQAM, (2008).

[56] M. Teboulle, Convergence of proximal-like algorithms, SIAM Journal on Optimization, 6 (1997), pp. 617-625.

[57] V. H. Vroom, Work and motivation, New York, Wiley, 1964. 